\title{
Correspondence
}

\section{Enigmatic death of an infant}

Sir,

The report of Hirschberger and Kleinberg (Archives, $1976,51,977)$ was stimulating; it is always a challenge to read about enigmatic cases. The authors describe an infant who failed to thrive and died at the age of $4 \frac{1}{2}$ months; on repeated occasions they found high urinary excretion of homovanillic acid (HVA) and vanillylmandelic acid (VMA). On microscopical examination of necropsy material the brain, lung, thyroid, liver, adrenal glands, and intestinal tract were normal. From their data (HVA and VMA, opsoclonus on one occasion, 'abnormal vertical eye movements'), even in the absence of a detailed report of neurological findings, we would like to suggest a diagnosis of myoclonic encephalopathy of infants (Kinsbourne, 1962).

Although failure to thrive, without accompanying diarrhoea, is not a usual symptom of myoclonic encephalopathy of infants, this could represent an age-specific manifestation. In their report the authors do not mention microscopical examination of the neural crest; therefore a possibly very small tumour (ganglioneuroblastoma) was not completely ruled out. However, even if the authors would agree with our diagnosis, there remains another enigma: as to the relationship between the hormones (HVA, VMA) and the clinical symptomatology-in this as well as in other patients. Nevertheless, in a future similar case, a therapeutic trial with ACTH would seem to be justified.

\section{P. KOEPP and U. BURCK, Universitätskrankenhaus, Eppendorf, Martinistr. 52, D-2000 Hamburg 20, BRD.}

Dr. Kleinberg comments:

We are grateful for the reference by Drs. Kopp and Burck to myoclonic encephalopathy, but hasten to point out that there are significant differences between our case and those presented in that report (Kinsbourne, 1962). Our case did not have myoclonic movements. There were occasional episodes of opisthotonic posturing and opsoclonus of the eyes, but myoclonus was simply not a feature of the neurological exam of our infant. Secondly, the youngest infant in Kinsbourne's series was 6 months old and almost all were considerably older. None presented with failure to thrive and none died. Lastly, of course, we are concerned about raised HVA and VMA levels which are not reported in Kinsbourne's paper.

\section{Reference}

Kinsbourne, M. (1962). Myoclonic encephalopathy of infants. Journal of Neurology, Neurosurgery and Psychiatry, 25, 271-276.
Sir,

I read with interest Hirschberger and Kleinberg's short report. There were some remarkable signs and symptoms in the infant described which I have personally noted in sudden infant death syndrome (SIDS) children; in fact I consider this infant a typical SIDS child.

In my studies of this syndrome I have come to the conclusion that the chief lesion in about $90 \%$ of SIDS children is a necrosis in the adenohypophysis or hypothalamus, caused by hypoxia or anoxia during fetal life, in cases of placental insufficiency. This finding was also reported by Sinclair Smith et al. (1976), who found retarded growth of the costochondral junctions of SIDS children. The child under discussion showed an absolute standstill in growth, which might have been caused by a lesion in the pituitary or hypothalamus. There was a reason for hypoxia during the mother's pregnancy (pneumonia in the seventh month). The lesions in the adenohypophysis have been imitated in rabbits by Lopes de Faria and Sokei (1973) by orthostatic collapse and were found by myself in an SIDS child (Visser, 1977). The authors do not mention examination of the pituitary at necropsy, nor the cerebral weight.

The neurological symptoms (opsoclonus and abnormal vertical eye movements) the infant showed during life point to a lesion of the brain stem described by Naeye (1976). He found astroglial proliferation in the brain stem combined with chromaffin hyperplasia in the adrenals in SIDS children. We found severe dislocation of the cerebellum and brain stem into the occipital foramen in an SIDS child of 11 months and considered it one of the causes of death in these children. In our case there was no cerebral oedema, yet the gyri of the cerebrum were flattened. The pituitary weighed $90 \mathrm{mg}$ (normal for this age at least $120 \mathrm{mg}$ ). The stalk of the pituitary also showed damage caused by extrinsic pressure.

How are these brain lesions caused? Growth disturbances in infants, caused by deficiency of growth hormone, involve the skeleton, muscle tissue, internal and endocrine organs, but not the brain. Skull growth is retarded and the effect is a progressive noncorrelation of the brain mass and skull volume. The effect is dolichocephaly (seen in the photograph of Hirschberger and Kleinberg's patient) and progressive cerebral compression, which are responsible for the cerebral lesions and the typical neurological symptoms during life. Another relevant point is that at necropsy there were no signs of infection in any organ, yet blood culture yielded pneumococci. We have also found this phenomenon in SIDS children.

How are the increased levels of HVA and VMA explained in the infant under discussion despite the lack of a neural crest tumour? The only endocrine organ which might be responsible for the production of both these catecholamines is the pineal gland. It produces catecholamines, histamine, serotonin, glomerulotrophin, and 
melanotrophin, and also has a close neural relationship with adrenal medullary tissue. There also seems to be a subtle balance between the hypothalamic/pituitary system and the pineal gland. If one of these (opposed) endocrine systems suffers a deficiency or lesion, the other will show hyperfunction. This could explain the increased HVA and VMA values in the child. We saw a similar case, but in a young man, showing increased VMA values and also operated on for a suspected pheochromocytoma, which was negative (Visser and Axt, 1975). Through morphometric techniques we proved a new clinicopathological entity, 'adrenal medullary hyperplasia', causing overproduction of catecholamines. The increased HVA values in the infant discussed were always higher than the VMA values, suggesting a combined hyperfunction of both pineal gland and adrenal medulla caused by hypopituitarism.

In conclusion, we expect that in the infant under discussion there existed (a) a lesion of the adenohypophysis, contracted in the seventh month of pregnancy by pneumonia and possible hypoxia of the mother; (b) subsequent hyperpinealism responsible for the production of HVA and VMA combined with hypertension (probably intermittent) during life; (c) (relatively) increased weight of cerebrum and heart; and (d) disturbed cortico/medullary volume ratios in the adrenals.

J. W. VISSER

Pathological Department, Laboratorium voor de

Volksgezondheid in Friesland, The Netherlands.

\section{References}

Lopes de Faria, J., and Sokei, E. L. (1973). Necrotic changes of the rabbit adenohypophysis following orthostatic collapse. Beitrage zur Pathologie, 150, 400-405.

Naeye, R. L. (1976). Brainstem and adrenal abnormalities in the sudden-infant-death syndrome. American Journal of Clinical Pathology, 66, 527-530.

Sinciair-Smith, C., Dinsdale, F., and Emery, J. (1976). Evidence of duration and type of illness in children found unexpectedly dead. Archives of Disease in Childhood, 41, $424-429$.

Visser, J. W. (1977). Sudden infant death syndrome and its probable cause. Journal of Clinical Pathology (in press).

Visser, J. W., and Axt, R. (1975). Bilateral adrenal medullayr hyperplasia; a clinicopathological entity. Journal of Clinical Pathology, 28, 298-304.

\section{Dr. Kleinberg comments:}

We are intrigued by Dr. Visser's suggestion that our child is in fact a 'typical SIDS child.' Such infants are usually considered to be normal, to be growing and developing normally, and are not believed to have lifethreatening illness before their unexpected death. Our child presented a marked failure to thrive and his death was neither unexpected nor sudden. We recognize, of course, that many SIDS infants have disturbances of autonomic cardiovascular systems and/or sleep states, but these abnormalities have been generally uncovered in chance observations upon clinically normal infants who later succumb to SIDS. Lastly, the pathologist commented that adrenals and pineal were unremarkable. Though the weight of the pituitary was unavailable, the brain weight was $460 \mathrm{~g}$.

FREDERIC KLEINBERG, Mayo Clinic, Rochester, Minn. 55901, USA.

\section{Treatment of severe Asian rickets with vitamin D-fortified chupatti flour}

Sir,

Vitamin D deficiency, leading to severe rickets and osteomalacia, is common among Asians in Britain (Dunnigan et al., 1962; Ford et al., 1972, 1976). The fortification of chupatti flour with vitamin $D$ has been shown to raise levels of serum 25-hydroxy-vitamin D and to reduce the incidence of biochemical rickets in those consuming it (Pietrek et al., 1976). We report here the successful use of the flour in a case of severe, untreated Asian rickets.

A 16-year-old Asian youth presented with a history of gradual bending of both legs which had become increasingly painful over the previous 6 months. There was moderate bowing of both tibiae. Serum $\mathrm{Ca}$ was 7.6 $\mathrm{mg} / 100 \mathrm{ml}(1.9 \mathrm{mmol} / \mathrm{l})$, phosphate $3.3 \mathrm{mg} / 100 \mathrm{ml}$ (1.06 mmol/l), and alkaline phosphatase $65 \mathrm{King}$ Armstrong units $/ 100 \mathrm{ml}$. Serum 25-hydroxy-vitamin D was low at $3.5 \mathrm{ng} / \mathrm{ml}$ (Belsey et al., 1974). Plasma proteins, urea, and electrolytes, D-xylose absorption, and faecal fats were normal. $X$-rays of wrists and knees showed the typical epiphyseal and metaphyseal changes of rickets. A jejunal biopsy was normal and an iliac crest bone biopsy showed bone trabeculae with thickened osteoid seams on their surfaces consistent with the diagnosis of osteomalacia (Dr. T. Anderson). He was considered to be a typical case of adolescent Asian rickets without evidence of renal disease or malabsorption.

The patient's family was supplied for 16 months with flour fortified with 6000 units vitamin $D_{2}$ per kilogram, prepared as described previously (Pietrek et al., 1976). The patient consumed 4 chupatties (about $160 \mathrm{~g}$ flour) daily containing approximately 1000 units vitamin $D_{2}$; about half the vitamin appears to be destroyed in cooking (Pietrek et al., 1976), providing him with an effective intake of about 500 units daily.

The biochemical response to the consumption of the flour is shown in the Fig. Serum $\mathrm{Ca}$ and alkaline phosphatase levels returned to normal after 7 months and serum 25-hydroxy-vitamin D levels rose to a peak of $22 \mathrm{ng} / \mathrm{ml}$ after 10 months and levelled out at about $18 \mathrm{ng} / \mathrm{ml}$. Radiological healing was complete at the end of the 16-month period and a second bone biopsy at this time showed complete histological healing.

This case report confirms that vitamin D-fortified chupatti flour is effective in the treatment of Asian rickets. Biochemical, radiological, and histological healing showed that the vitamin D preparation used retains its biological 